\title{
Robotic-Assisted Transanal Repair of a Rectovaginal Fistula
}

\author{
Melinda E. Stack ${ }^{1} \cdot$ Konstantin Umanskiy ${ }^{1}$
}

Received: 18 April 2016 / Accepted: 9 May 2016/Published online: 24 May 2016

(C) 2016 The Society for Surgery of the Alimentary Tract

\begin{abstract}
Background Various methods exist for the treatment of rectovaginal fistulas, with the choice of repair largely dependent on the fistula location. Options include local repair with mucosal advancement flaps, the use of agents like fibrin glue, and an abdominal approach with resection and colo-anal reconstruction. Traditionally, local repair with mucosal advancement flaps is reserved for simple, low rectovaginal fistulas, while high rectal fistulas require a transabdominal approach. ${ }^{1,2}$

Methods Here, we demonstrate an innovative approach for the treatment of a complex, high rectovaginal fistula in a patient with a hostile pelvis via a transanal approach with robotic assistance. The video demonstrates the basic steps of a repair of a rectovaginal fistula: debridement of the fistula tract, mobilization of the mucosal advancement flap, primary closure of the fistula tract, suturing of the mucosal advancement flap, and flexible sigmoidoscopy to confirm lumen patency and visualization of the closure.

Results By utilizing robotic assistance, we were provided with improved dexterity, precision, and scalability to accomplish the complex task of dissection, suturing, and knot tying in the confines of the rectum.

Conclusions We suggest that this approach can be used on selected patients with high rectovaginal fistula or other rectal pathology who are otherwise not candidates for a transanal approach.
\end{abstract}

Keywords Rectovaginal fistula $\cdot$ Robotic surgical procedures $\cdot$ Colorectal surgery

Presented as a video presentation at the SSAT 57th Annual Meeting, May 22nd, 2016.

Electronic supplementary material The online version of this article (doi:10.1007/s11605-016-3168-1) contains supplementary material, which is available to authorized users.

Konstantin Umanskiy

kumanskiy@surgery.bsd.uchicago.edu

1 Department of Surgery, The University of Chicago Medicine, 5841 S. Maryland Ave., MC5095, Chicago, IL 60637, USA
Author Contribution Each author significantly contributed to the concept and design of this project, video editing and writing of the manuscript.

Compliance with Ethical Standards

Grant Support None

\section{References}

1. Rivadeneira DE, Ruffo B, Amrani S, Salinas C. Rectovaginal fistulas: Current surgical management. Clinics in Colon and Rectal Surgery 2007;20:96-101.

2. Rothenberger DA, Christenson CE, Balcos EG, Schottler JL, Nemer FD, Nivatvongs S, Goldberg SM. Endorectal advancement flap for treatment of simple rectovaginal fistula. Diseases of the Colon and Rectum 1982;25(4):297-300. 